\title{
Landscape solution to the SUSY flavor and $C P$ problems
}

\author{
Howard Baer $\odot,{ }^{1, *}$ Vernon Barger, ${ }^{2, \dagger}$ and Dibyashree Sengupta $\odot^{1, \sharp}$ \\ ${ }^{1}$ Homer L. Dodge Department of Physics and Astronomy, University of Oklahoma, Norman, Oklahoma 73019, USA \\ ${ }^{2}$ Department of Physics, University of Wisconsin, Madison, Wisconsin 53706, USA
}

(Received 6 October 2019; published 16 December 2019)

\begin{abstract}
In a fertile patch of the string landscape which includes the minimal supersymmetric standard model (MSSM) as the low-energy effective theory, rather general arguments from Douglas suggest a power-law statistical selection of soft breaking terms [ $m_{\text {soft }}^{n}$ where $n=2 n_{F}+n_{D}-1$ with $n_{F}$ the number of hidden sector $F$-supersymmetry (SUSY) breaking fields and $n_{D}$ the number of $D$-term SUSY breaking fields]. The statistical draw towards large soft terms must be tempered by requiring an appropriate breakdown of electroweak (EW) symmetry with no contributions to the weak scale larger than a factor 2-5 of its measured value, lest one violates the (anthropic) atomic principle. Such a simple picture of stringy naturalness generates a light Higgs boson with mass $m_{h} \simeq 125 \mathrm{GeV}$ with sparticles (other than higgsinos) typically beyond LHC reach. Then we expect first and second generation matter scalars to be drawn independently to the tens of $\mathrm{TeV}$ regime where the upper cutoff arises from two-loop renormalization group equation (RGE) terms that drive third generation soft masses towards tachyonic values. Since the upper bounds on $m_{0}(1,2)$ are the same for each generation, and flavor independent, then these will be drawn toward quasidegenerate values. This mechanism leads to a natural mixed decoupling/quasidegeneracy solution to the SUSY flavor problem and a decoupling solution to the SUSY $C P$ problem.
\end{abstract}

DOI: 10.1103/PhysRevResearch.1.033179

\section{INTRODUCTION}

The emergence of the string landscape picture [1-5] provides so far the only plausible mechanism for understanding the extreme suppression of the vacuum energy density of the universe $\rho_{v a c}=\Lambda c^{2} /\left(8 \pi G_{N}\right) \simeq(3 \mathrm{meV})^{4}$ from its expected value $\sim m_{P}^{4}$ (over 120 orders of magnitude suppression). Assuming a multiverse [6] with a huge (of order $10^{500}$ [7] or far greater? [8]) assortment of vacua states with cosmological constant (CC) uniformly distributed across the decades, then those pocket universes with $\Lambda$ somewhat larger than our measured value would lead to such rapid expansion that galaxies would not condense, and presumably observers would not arise. Weinberg used such reasoning to predict the value of $\Lambda$ to within a factor of several well before it was experimentally measured $[9,10]$.

Given the success of the landscape in predicting $\Lambda$, can multiverse arguments also be used to predict the scale of supersymmetry (SUSY) breaking [11,12]? A statistical approach to understanding the SUSY breaking scale has been advocated by Douglas $[12,13]$. In this approach, naturalness is replaced by stringy naturalness $[14,15]$ wherein observable $\mathcal{O}_{2}$ is more natural than observable $\mathcal{O}_{1}$ if more phenomeno-

\footnotetext{
*baer@ou.edu

†barger@pheno.wisc.edu

†ibyashree.Sengupta-1@ou.edu
}

Published by the American Physical Society under the terms of the Creative Commons Attribution 4.0 International license. Further distribution of this work must maintain attribution to the author(s) and the published article's title, journal citation, and DOI. logically viable vacua lead to $\mathcal{O}_{2}$ than to $\mathcal{O}_{1}$. The key phrase "phenomenologically viable" can be used here in an anthropic sense, as in the case of the cosmological constant, in that such vacua lead to pocket universes that can admit life as we understand it. [12]

Specifically, we might write the distribution of vacua as

$$
\begin{aligned}
& d N_{\text {vac }}\left[m_{\text {hidden }}^{2}, m_{\text {weak }}, \Lambda\right] \\
& \quad=f_{\text {SUSY }}\left(m_{\text {hidden }}^{2}\right) \cdot f_{\mathrm{EWSB}} \cdot f_{\mathrm{CC}} \cdot d m_{\text {hidden }}^{2}
\end{aligned}
$$

where $m_{\text {hidden }}$ is a mass scale associated with hidden sector SUSY breaking which gives rise to (in gravity mediation, which is assumed here) ${ }^{1}$ a gravitino mass $m_{3 / 2} \simeq m_{\text {hidden }}^{2} / m_{P}$ via the super-Higgs mechanism. In such models, then we expect the appearance of soft SUSY breaking terms, collectively denoted here as $m_{\text {soft }}$, of order $m_{\text {soft }} \sim m_{3 / 2}$ [22-24].

For the prior distribution $f_{\text {SUSY }}$, Douglas proposed on rather general grounds a power-law ansatz $[11,12]$

$$
f_{\text {SUSY }}\left(m_{\text {hidden }}^{2}\right) \sim\left(m_{\text {hidden }}^{2}\right)^{2 n_{F}+n_{D}-1},
$$

where $n_{F}$ is the number of hidden sector $F$-breaking fields and $n_{D}$ is the number of contributing $D$-breaking fields. This

\footnotetext{
${ }^{1}$ In gauge mediation, typically the trilinear $A$ parameter $\sim 0$ so there is little mixing in the stop sector, and consequently too light a value for the SM-like Higgs boson $m_{h}$, unless soft terms have extremely large, unnatural values [16-19]. In gravity mediation, since then we expect large $A$ terms, there is no such problem to gain $m_{h} \simeq 125 \mathrm{GeV}$ with natural soft term values under the $\Delta_{\text {EW }}$ fine-tuning measure [20,21].
} 
is reflective of general string theory models which typically contain of order 10 hidden sectors some or all of which might contribute to SUSY breaking. Only for $n_{F}=0, n_{D}=1$ would we obtain (the usually assumed) uniform distribution of soft breaking terms. Already for $n_{D}=0, n_{F}=1$, we would expect a linear statistical draw towards large soft terms. For more complicated hidden sectors, then the statistical draw toward large soft terms would be even stronger.

Early on, these considerations led to extensive debate over whether to expect high scale or weak scale SUSY breaking $[11,12,25]$. Such debate was in part predicated on the influence of cosmological constant selection on the SUSY breaking scale. Initial expectations were that $f_{\mathrm{CC}} \sim \Lambda / m_{\text {hidden }}^{4}$. Following Douglas [12], the consensus emerged that $f_{\mathrm{CC}}$ would be independent of the SUSY breaking sector, and that $f_{\mathrm{CC}} \sim$ $\Lambda / m_{\text {string }}^{4}$.

The third element in Eq. (1) is $f_{\text {EWSB }}$. This function contains any anthropic requirements. For the case of SUSY, it also depends on the anticipated solution to the SUSY $\mu$ problem: why is the SUSY conserving $\mu$ parameter of order the weak scale rather than the Planck scale [26]? Here, we will assume a natural solution to the SUSY $\mu$ problem, i.e., that $|\mu| \sim m_{\text {weak }}$. If $|\mu| \gg m_{\text {weak }}$, then some fine-tuning would be required to gain a value of $m_{\text {weak }}$ close to the $100-\mathrm{GeV}$ scale. Such fine-tuning requires a tiny range of compensating opposite-sign soft terms to maintain the weak scale not-toofar from its measured value [15]. And as shown by nuclear physics calculations of Agrawal et al. [27], a pocket universe value of $m_{\text {weak }}$ displaced by a factor 2-5 from our measured value would lead to catastrophes in nuclear physics that would violate the atomic principle.

The magnitude of the weak scale is related to SUSY Lagrangian parameters via the scalar potential minimization condition

$$
\begin{aligned}
m_{Z}^{2} / 2 & =\frac{m_{H_{d}}^{2}+\Sigma_{d}^{d}-\left(m_{H_{u}}^{2}+\Sigma_{u}^{u}\right) \tan ^{2} \beta}{\tan ^{2} \beta-1}-\mu^{2} \\
& \simeq-m_{H_{u}}^{2}-\Sigma_{u}^{u}-\mu^{2},
\end{aligned}
$$

where the $\Sigma_{u}^{u}$ and $\Sigma_{d}^{d}$ terms contain a large sum of radiative corrections (for expressions, see Appendix to Ref. [21]). In fact, the electroweak fine-tuning measure $\Delta_{\mathrm{EW}}[20,21]$ conservatively requires that the weak scale terms on the righthand side of Eq. (3) be comparable to the observed value $m_{Z}^{2} / 2$ on the left-hand-side. This is a manifestation of the notion of practical naturalness [28]: that various additive contributions to any observable should be comparable to or less than that observable: if not, then (implausible) fine-tunings are required to enforce the observable at its measured value. In most SUSY phenomenology papers, the measured value of $m_{Z}$ is used to fix (fine-tune) the value of $\mu^{2}$. In our approach, since $\mu$ is already fixed at a natural value due to the solution to the SUSY $\mu$ problem, then $m_{Z}$ is left variable. We denote the various pocket universe values of the $Z$ mass as $m_{Z}^{\mathrm{PU}}$ which is different from the measured value in our universe [29,30]. With $\mu$ fixed, then different statistical entries for the soft terms will determine an associated pocket universe value for the weak scale, and consequently for $m_{Z}^{\mathrm{PU}}$.

An initial guess for this term [31] was $f_{\text {EWSB }} \sim$ $\left(m_{\text {weak }} / m_{\text {soft }}\right)^{2}$ which follows the gross behavior of fine-tuning measures $\Delta_{\mathrm{BG}}$ [32] ( $\left.\Delta_{\mathrm{EW}}\right)$ which compare the largest high scale (weak scale) SUSY breaking contribution to the size of the weak scale itself: then the ansatz for $f_{\mathrm{EWSB}}$ rewards vacua with soft terms that are closest to the magnitude of the weak scale itself.

As pointed out in Refs. [29,30], this ansatz fails in five cases (and a sixth case will be discussed later in Sec. II). (1) Very large trilinear soft terms lead to charge-or-color breaking (CCB) vacua. Such vacua are unlikely to support the existence of atoms ${ }^{2}$ (the atomic principle) and hence life as we know it. Such CCB minima must be vetoed and not merely penalized by a statistical factor.

(2) If other soft terms such as $m_{H_{u}}^{2}$ are too large, then they are not driven negative at $Q=m_{\text {weak }}$ and EW symmetry is not even broken. Such vacua must also be vetoed.

(3) For small (seemingly more natural) values of $m_{H_{u}}^{2}$, then $m_{H_{u}}^{2}$ is driven to large negative values, resulting in too large of values of $m_{Z}^{\mathrm{PU}}$, in violation of Agrawal et al. limits. As $m_{H_{u}}^{2}(\Lambda)$ increases, its weak scale value decreases (radiatively driven naturalness) resulting in a more natural theory with $m_{Z}^{\mathrm{PU}}$ close to the measured value in our universe.

(4) As the trilinear soft term $A_{0}$ increases (seemingly more unnatural), then large cancellations in $\Sigma_{u}^{u}\left(\tilde{t}_{1,2}\right)$ render these contributions more natural, and $m_{Z}^{\mathrm{PU}}$ closer to our measured value.

(5) Even in the event of appropriate EW symmetry breaking, the ansatz $f_{\text {EWSB }} \sim\left(m_{\text {weak }} / m_{\text {soft }}\right)^{2}$ penalizes but does not forbid vacua with too large a value of $m_{\mathrm{Z}}^{\mathrm{PU}}$. Surviving vacua with $m_{Z}^{\mathrm{PU}} \gtrsim(2-5) m_{Z}^{\text {meas }}$ must be vetoed since these would contradict the nuclear physics analyses of Agrawal et al. [27].

To ameliorate this situation, it was proposed in Refs. $[29,30]$ to instead veto any non-standard EW vacua and also to veto any vacua with too large a value of $m_{Z}^{\mathrm{PU}}$ greater than a factor four larger than our measured value. For a fixed natural value of $\mu$, this latter condition corresponds to vetoing pocket universes with $\Delta_{\mathrm{EW}}>30$. Thus we also implement

$$
f_{\text {EWSB }}=\Theta\left(30-\Delta_{\text {EW }}\right) .
$$

By scanning over models such as NUHM2 [33] or NUHM3 which allow for an input $\mu$ parameter, with soft terms generated according to $m_{\text {soft }}^{n}$ for $n=1$ and 2, along with the anthropic vetos from $f_{\mathrm{EWSB}}$, then the following features were found [30].

(1) A statistical peak was found at $m_{h} \simeq 125 \pm 2 \mathrm{GeV}$. This is easy to understand: we are selecting for soft terms as large as possible subject to appropriate EWSB and a value of $m_{Z}^{\mathrm{PU}} \lesssim 4 m_{Z}^{\text {meas }}$. This also selects for large (but not so large as to lead to CCB minima) $A_{0}$ terms which increase top squark mixing and lift $m_{h}$ up to the vicinity of $125 \mathrm{GeV}$. (2) The probability distribution $d P / d m_{\tilde{g}}$ yields a value $m_{\tilde{g}} \sim 4 \pm 2$ $\mathrm{TeV}$, safely above LHC2 limits. (3) The light top squark is lifted to $m_{\tilde{t}_{1}} \sim 1.5 \pm 0.5 \mathrm{TeV}$, also safely above LHC Run 2 limits. (4) Light higgsinos $\widetilde{\chi}_{1}^{ \pm}$and $\widetilde{\chi}_{1,2}^{0}$ with mass $\sim \mu \sim$ $200 \pm 100 \mathrm{GeV}$. The mass gap is $m_{\widetilde{\chi}_{2}^{0}}-m_{\widetilde{\chi}_{1}^{0}} \sim 7 \pm 3 \mathrm{GeV}$.

\footnotetext{
${ }^{2}$ This is known as the atomic principle: life as we know it seems to require the existence of atoms and molecules as exist in, e.g., our universe.
} 
Thus higgsino pair production signals should ultimately show up at LHC14 via $p p \rightarrow \widetilde{\chi}_{1}^{0} \widetilde{\chi}_{2}^{0}$ production followed by $\tilde{\chi}_{2}^{0} \rightarrow$ $\ell^{+} \ell^{-} \tilde{\chi}_{1}^{0}$ decay with $m\left(\ell^{+} \ell^{-}\right)<(7 \pm 3) \mathrm{GeV}$ once sufficient luminosity is gained [34-36]. (5) First and second generation matter scalars (squarks and sleptons) are pulled up to $m(\tilde{q}, \tilde{\ell}) \sim 20 \pm 10 \mathrm{TeV}$.

The present paper focuses on this latter point. Apparently, with first and second generation matter scalars being pulled up to the multi-TeV regime, then one is also being pulled up to a potential decoupling solution to the SUSY flavor and $C P$ problems. The question is: how does this decoupling arise, and is it enough to actually solve these two SUSY issues?

\section{LIVING DANGEROUSLY WITH HEAVY SFERMIONS}

In Sec. I, we emphasized that Douglas' general stringy considerations imply a statistical draw towards large soft terms. However, the soft terms cannot become arbitrarily large without leading to nonstandard $\mathrm{EW}$ vacua or else too large of a value of pocket universe weak scale $m_{Z}^{\text {PU }}$ : such vacua must be anthropically vetoed.

Here, we concern ourselves with the upper bound on matter sfermion masses for the first two generations, which we label according to high-scale soft term values $m_{0}(1)$ and $m_{0}(2)$. For simplicity, we will assume all high scale matter sfermion masses within a single generation are degenerate [as is expected in models containing some remnant $S O(10)$ GUT symmetry]. These could be placed for context within the $i$-extra parameter nonuniversal Higgs models [33] (NUHMi, $i=2-4)$. In NUHM2, $m_{0}(1)=m_{0}(2)=m_{0}(3)$, while in NUHM3, $m_{0}(1)=m_{0}(2) \neq m_{0}(3)$. Here, NUHM4 is considered since we are allowing for splittings between first and second generation masses (as well as the third), i.e., $m_{0}(1) \neq$ $m_{0}(2) \neq m_{0}(3)$. But we will also allow for the presence of off-diagonal soft term masses. To make contact with general constraints from SUSY flavor and $C P$-violating processes, as presented, for instance, in Refs. [37-41]. we will work within the superCKM mass basis wherein the quark and lepton mass matrices are diagonal but the squark and slepton mass matrices are not yet diagonalized.

From a scan over NUHM3 parameter space in Ref. [30], it was found that the statistical distribution of first/second generation sfermion masses for $n=1$ or 2 was peaked around $m_{\tilde{f}} \sim 20 \mathrm{TeV}$ but with tails extending as far as $40 \mathrm{TeV}$. What sets the upper bound for such sfermion masses?

At first sight, the $\Sigma_{u}^{u}$ and $\Sigma_{d}^{d}$ terms contain first/second generation $D$-term contributions to the EW scale. For first/second generation sfermions, neglecting the small Yukawa couplings, we find the contributions

$$
\Sigma_{u, d}^{u, d}\left(\tilde{f}_{L, R}\right)=\mp \frac{c_{\mathrm{col}}}{16 \pi^{2}} F\left(m_{\tilde{f}_{L, R}}^{2}\right)\left(-4 g_{Z}^{2}\left(T_{3}-Q_{\mathrm{em}} x_{W}\right)\right),
$$

where $T_{3}$ is the weak isospin, $Q_{\mathrm{em}}$ is the electric charge assignment (taking care to flip the sign of $Q_{\mathrm{em}}$ for right-sfermions), $c_{\text {col }}=1$ (3) for color singlet (triplet) states, $x_{W} \equiv \sin ^{2} \theta_{W}$ and where

$$
F\left(m^{2}\right)=m^{2}\left(\ln \frac{m^{2}}{Q^{2}}-1\right) .
$$

We adopt an optimized scale choice $Q^{2}=m_{\text {SUSY }}^{2} \equiv m_{\tilde{t}_{1}} m_{\tilde{t}_{2}}{ }^{3}$ The explicit first generation squark contributions to $\Sigma_{u}^{u}$ (neglecting the tiny Yukawa couplings) are given by

$$
\begin{aligned}
& \Sigma_{u}^{u}\left(\tilde{u}_{L}\right)=\frac{3}{16 \pi^{2}} F\left(m_{\tilde{u}_{L}}^{2}\right)\left[-4 g_{Z}^{2}\left(\frac{1}{2}-\frac{2}{3} x_{W}\right)\right], \\
& \Sigma_{u}^{u}\left(\tilde{u}_{R}\right)=\frac{3}{16 \pi^{2}} F\left(m_{\tilde{u}_{R}}^{2}\right)\left[-4 g_{Z}^{2}\left(\frac{2}{3} x_{W}\right)\right], \\
& \Sigma_{u}^{u}\left(\tilde{d}_{L}\right)=\frac{3}{16 \pi^{2}} F\left(m_{\tilde{d}_{L}}^{2}\right)\left[-4 g_{Z}^{2}\left(-\frac{1}{2}+\frac{1}{3} x_{W}\right)\right], \\
& \Sigma_{u}^{u}\left(\tilde{d}_{R}\right)=\frac{3}{16 \pi^{2}} F\left(m_{\tilde{d}_{R}}^{2}\right)\left[-4 g_{Z}^{2}\left(-\frac{1}{3} x_{W}\right)\right] .
\end{aligned}
$$

These contributions, arising from electroweak $D$-term contributions to masses, are frequently neglected since the various contributions cancel amongst themselves in the limit of mass degeneracy due to the fact that weak isospins and electric charges (or weak hypercharges) sum to zero in each generation. However, if squark and slepton masses are in the multi$\mathrm{TeV}$ regime but are nondegenerate within each generation, then the contributions may be large and noncancelling. In this case, they may render a theory which is otherwise considered to be natural, in fact, unnatural.

The first generation slepton contributions to $\Sigma_{u}^{u}$ are given by

$$
\begin{aligned}
& \Sigma_{u}^{u}\left(\tilde{e}_{L}\right)=\frac{1}{16 \pi^{2}} F\left(m_{\tilde{e}_{L}}^{2}\right)\left[-4 g_{Z}^{2}\left(-\frac{1}{2}+x_{W}\right)\right], \\
& \Sigma_{u}^{u}\left(\tilde{e}_{R}\right)=\frac{1}{16 \pi^{2}} F\left(m_{\tilde{e}_{R}}^{2}\right)\left[-4 g_{Z}^{2}\left(-x_{W}\right)\right], \\
& \Sigma_{u}^{u}\left(\tilde{v}_{L}\right)=\frac{1}{16 \pi^{2}} F\left(m_{\tilde{v}_{e L}}^{2}\right)\left[-4 g_{Z}^{2}\left(\frac{1}{2}\right)\right],
\end{aligned}
$$

these may also be large for large $m_{\tilde{\ell}}^{2}$ although again they cancel amongst themselves in the limit of slepton mass degeneracy.

In our evaluation of $\Delta_{\mathrm{EW}}$, in fact we sum all contributions from a complete generation before including them into $\Delta_{\mathrm{EW}}$. This allows for complete $D$-term cancellations in the limits of weak scale sfermion degeneracy. Of course, the sfermions are not completely degenerate at the weak scale even if they begin as degenerate at the high scale $Q \equiv m_{\mathrm{GUT}}$ due at least to weak scale $D$-term contributions to their masses. We have evaluated these contributions and find they lead to upper bounds on $m_{0}(1,2) \lesssim 5000 \mathrm{TeV}$ for $\Delta_{\mathrm{EW}}<30$, so that these $D$ terms do not set the upper limits on first/second generation sfermion masses.

A stricter constraint on first/second generation sfermion masses from the landscape comes from 2-loop renormalization group equation (RGE) contributions to the running of sfermion masses. The form of the two loop RGEs for sfermion

\footnotetext{
${ }^{3}$ The optimized scale choice is chosen to minimize the logarithmic contributions to $\Sigma_{u}^{u}\left(\tilde{t}_{1,2}\right)$ which occur to all orders in perturbation theory.
} 
masses is given by

$$
\frac{d m_{i}^{2}}{d t}=\frac{1}{16 \pi^{2}} \beta_{m_{i}^{2}}^{(1)}+\frac{1}{\left(16 \pi^{2}\right)^{2}} \beta_{m_{i}^{2}}^{(2)},
$$

where $t=\ln Q, i=Q_{j}, U_{j}, D_{j}, L_{j}$, and $E_{j}$, and $j=1-3$ is a generation index. The one loop $\beta$ function for the evolution of third generation scalar masses depends only on third generation and Higgs scalar masses and on the gaugino masses. The two loop terms are formally suppressed relative to one loop terms by the square of a coupling constant as well as an additional loop factor of $16 \pi^{2}$. However, these two loop terms include contributions from all scalars. Specifically, the two loop $\beta$ functions include [42]

$$
\beta_{m_{i}^{2}}^{(2)} \ni a_{i} g_{3}^{2} \sigma_{3}+b_{i} g_{2}^{2} \sigma_{2}+c_{i} g_{1}^{2} \sigma_{1},
$$

where

$$
\begin{aligned}
\sigma_{1}= & \frac{1}{5} g_{1}^{2}\left\{3\left(m_{H_{u}}^{2}+m_{H_{d}}^{2}\right)\right. \\
& \left.+\operatorname{Tr}\left[\mathbf{m}_{Q}^{2}+3 \mathbf{m}_{L}^{2}+8 \mathbf{m}_{U}^{2}+2 \mathbf{m}_{D}^{2}+6 \mathbf{m}_{E}^{2}\right]\right\}, \\
\sigma_{2}= & g_{2}^{2}\left\{m_{H_{u}}^{2}+m_{H_{d}}^{2}+\operatorname{Tr}\left[3 \mathbf{m}_{Q}^{2}+\mathbf{m}_{L}^{2}\right]\right\}
\end{aligned}
$$

and

$$
\sigma_{3}=g_{3}^{2} \operatorname{Tr}\left[2 \mathbf{m}_{Q}^{2}+\mathbf{m}_{U}^{2}+\mathbf{m}_{D}^{2}\right]
$$

and the $\mathbf{m}_{i}^{2}$ are squared mass matrices in generation space. The numerical coefficients $a_{i}, b_{i}$, and $c_{i}$ are related to the quantum numbers of the scalar fields, but are all positive quantities.

Thus, incorporation of multi-TeV masses for the first and second generation scalars leads to an overall positive, possibly dominant, contribution to the slope of third generation soft mass trajectories versus energy scale. Although formally a two loop effect, the smallness of the couplings is compensated by the much larger values of masses of the first two generations of scalars. In running from $m_{\mathrm{GUT}}$ to $m_{\text {weak }}$, this results in an overall reduction in third generation scalar masses. In fact, this effect was argued in Ref. [43] to lead to violation of naturalness constraints from a decoupling solution to the SUSY flavor problem. It was also used in Refs. [44,45] to generate SUSY models with an inverted scalar mass hierarchy to reconcile naturalness with a decoupling solution to the SUSY flavor and $C P$ problems along the lines of "effective supersymmetry" [46]. For values of sfermion masses which fall short of tachyonic, a sort of see-saw effect amongst scalar masses occurs: the higher the value of first and second generation scalar masses, the larger will be the two loop suppression of third generation and Higgs scalar masses. In this class of models, first and second generation scalars with masses of order 10-40 TeV may coexist with $\mathrm{TeV}$-scale third generation scalars, thus giving a very large suppression to both FCNC and $C P$ violating processes while driving third generation sfermions to natural values.

In the context of our string landscape picture, this is yet another example of living dangerously, ${ }^{4}$ wherein soft terms are

\footnotetext{
${ }^{4}$ Arkani-Hamed and Dimopoulos [47] state: "anthropic reasoning leads to the conclusion that we live dangerously close to violating
}

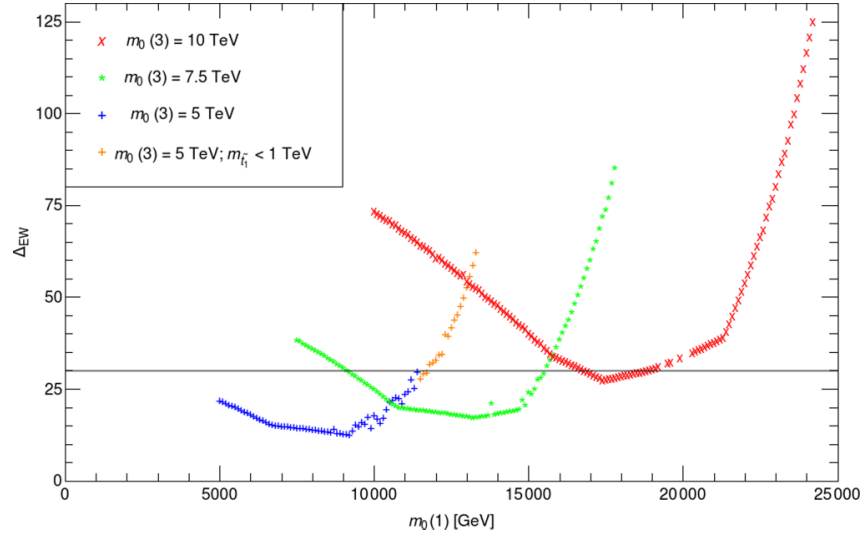

FIG. 1. We plot the value of $\Delta_{\text {EW }}$ vs $m_{0}(1,2)$ for $m_{0}(3)=5,7.5$, and $10 \mathrm{TeV}$ and $m_{1 / 2}=1200 \mathrm{GeV}, A_{0}=-1.6 m_{0}(3)$ and $\tan \beta=10$ with $\mu=200 \mathrm{GeV}$ and $m_{A}=2000 \mathrm{GeV}$.

pulled to large values which actually increases the naturalness of the theory so long as we stop short of impending disaster: which in this case would be that huge first/second generation sfermion masses might drive third generation masses tachyonic leading to $\mathrm{CCB}$ vacua.

The situation is illustrated in Fig. 1 where we adopt the NUHM3 model to plot the value of $\Delta_{\mathrm{EW}}$ versus $m_{0}(1,2)$ for $m_{1 / 2}=1200 \mathrm{GeV}, A_{0}=-1.6 m_{0}$ (3) and $\tan \beta=10$ with $\mu=$ $200 \mathrm{GeV}$ and $m_{A}=2000 \mathrm{GeV}$. We also take $m_{0}(3)=5,7.5$, and $10 \mathrm{TeV}$ (blue/orange, green and red curves, respectively). From the plot we see that as $m_{0}(1,2)$ increases, the models are driven to greater naturalness in that third generation soft terms are driven to smaller values by large two-loop RGE contributions. As $m_{0}(1,2)$ increases even further, then cancellations with the $\Sigma_{u}^{u}\left(\tilde{t}_{1,2}\right)$ terms are disrupted and the models again become more unnatural, leading to too large of contributions to the pocket universe weak scale $m_{Z}^{\mathrm{PU}}$. For even higher $m_{0}(1,2)$ values, then the top squark soft term $m_{\tilde{t}_{R}}^{2}$ is driven tachyonic leading to $\mathrm{CCB}$ vacua.

An important point is that for particular parameter values, we do gain an upper bound on first/second generation soft terms. The upper bound changes within parameter space variation, but depends only on gauge quantum numbers, so it is the same for both generations one and two. Thus, the first and second generation soft masses are pulled to large values by the landscape, but with the same upper bounds. This means that for strong enough pull, then $m_{0}(1)$ and $m_{0}(2)$ will be pulled to similar upper limits. If the pull is strong enough, they will be pulled towards quasi-degeneracy, which helps, along with decoupling, to solve the SUSY flavor problem.

\section{SUSY FLAVOR AND CP PROBLEMS}

\section{A. Flavor}

In the SM, a fourth quark, charm, was posited in order to suppress flavor changing neutral current (FCNC) processes, for which there were strict limits [48]. In a successful applica-

an important but fragile feature of the low-energy world...," in this case, appropriate electroweak symmetry breaking. 
tion of practical naturalness, Gaillard and Lee [49] required the charm-quark box diagram contribution to the $m_{K_{L}}-$ $m_{K_{S}} \equiv \Delta m_{K}$ mass difference to be less than the measured value of $\Delta m_{K}$ itself: this lead to the successful prediction that $1 \mathrm{GeV}<m_{c}<2 \mathrm{GeV}$ shortly before the charm quark discovery.

By supersymmetrizing the SM into the MSSM, then many new parameters are introduced, mainly in the soft SUSY breaking sector [50]. These include sfermion mass matrices

$$
\mathcal{L}_{\text {soft }} \ni-\tilde{f}_{i}^{\dagger}\left(\mathbf{m}_{\mathbf{f}}^{\mathbf{2}}\right)_{i j} \tilde{f}_{j},
$$

where $i$ and $j$ are generation indices $i, j=1-3$ and the sfermion index $\tilde{f}$ runs over the various matter superfields $\hat{Q}$, $\hat{U}^{c}, \hat{D}^{c}, \hat{L}^{c}$, and $\hat{E}^{c}$ in the notation of Ref. [51]. There are also trilinear soft terms that can contribute to flavor violation:

$$
\begin{aligned}
\mathcal{L}_{\text {soft }} \ni & \left(\mathbf{a}_{u}\right)_{i j} \epsilon_{a b} \tilde{Q}_{i}^{a} H_{u}^{b} \tilde{u}_{R j}^{\dagger}+\left(\mathbf{a}_{d}\right)_{i j} \tilde{Q}_{i}^{a} H_{d a} \tilde{d}_{R j}^{\dagger} \\
& +\left(\mathbf{a}_{e}\right)_{i j} \tilde{L}_{i}^{a} H_{d a} \tilde{e}_{R j}^{\dagger}+\text { H.c. }
\end{aligned}
$$

In gravity mediation, the trilinears are expected to be proportional to the corresponding Yukawa couplings so that these terms are small for first/second generation values. We will thus focus mainly on the mass matrices in Eq. (11).

In the superCKM basis, the $6 \times 6$ sfermion mass matrices are built out of $3 \times 3 L L, R R, L R$, and $R L$ submatrices which have the form, e.g., 5

$$
\left(\mathbf{m}_{\tilde{f}}^{2}\right)_{L L}=\left(\begin{array}{lll}
\left(m_{f 1}^{2}\right)_{L L} & \left(\Delta_{12}^{f}\right)_{L L} & \left(\Delta_{13}^{f}\right)_{L L} \\
\left(\Delta_{21}^{f}\right)_{L L} & \left(m_{f 2}^{2}\right)_{L L} & \left(\Delta_{23}^{f}\right)_{L L} \\
\left(\Delta_{31}^{f}\right)_{L L} & \left(\Delta_{32}^{f}\right)_{L L} & \left(m_{f 3}^{2}\right)_{L L}
\end{array}\right)
$$

with $\left(m_{\tilde{U}}^{2}\right)_{L L}=V_{L}^{u} \mathbf{m}_{Q}^{2} V_{L}^{u \dagger},\left(m_{\tilde{U}}^{2}\right)_{R R}=V_{R}^{u} \mathbf{m}_{U}^{2 T} V_{R}^{u \dagger},\left(m_{\tilde{U}}^{2}\right)_{L R}=$ $-\frac{v \sin \beta}{\sqrt{2}} V_{L}^{u} \mathbf{a}_{U}^{*} V_{R}^{u \dagger}$, etc. and where the CKM matrix is given by $V_{K M}=V_{L}^{u} V_{L}^{d \dagger}$. For mass matrices proportional to the unit matrix $\mathbf{m}_{\tilde{f}}^{2}=m_{\tilde{f}}^{2} \mathbf{1}$ (flavor universality), then no flavorchanging transitions are allowed and the SUSY flavor problem is solved. However, for gravity mediation, no known principles enforce flavor universality because the transformation that diagonalizes the quark mass matrices does not simultaneously diagonalize the corresponding squark mass squared matrices. In that case, then the off-diagonal mass matrix contributions $\Delta_{i j}^{f}$ may contribute to FCNC processes via mass insertions, and furthermore, nondegenerate diagonal terms can also lead to FCNC effects [52]. Constraints on the off-diagonal terms are typically listed in terms of dimensionless quantities $\left(\delta_{i j}^{f}\right)_{L L, R R, L R, R L} \equiv \frac{\left(\Delta_{i j}^{f}\right)_{L L, R R, L R, R L}}{\tilde{m}^{2}}$, where the $\tilde{m}$ represent an averaged sfermion mass for the corresponding mass matrix.

First we concentrate on limits for flavor-changing offdiagional mass matrix elements as they vary from the weak scale on into the decoupling regime. In Fig. 2, we list the most restrictive limits on several $\Delta_{i j}$ quantities arising from $\Delta m_{K}$ constraint [53-55] and also from updated branching fraction limits on $\mu \rightarrow e \gamma$ decay: $B F(\mu \rightarrow e \gamma)<4.2 \times 10^{-13}$

${ }^{5}$ For a more detailed review, see Ref. [41].

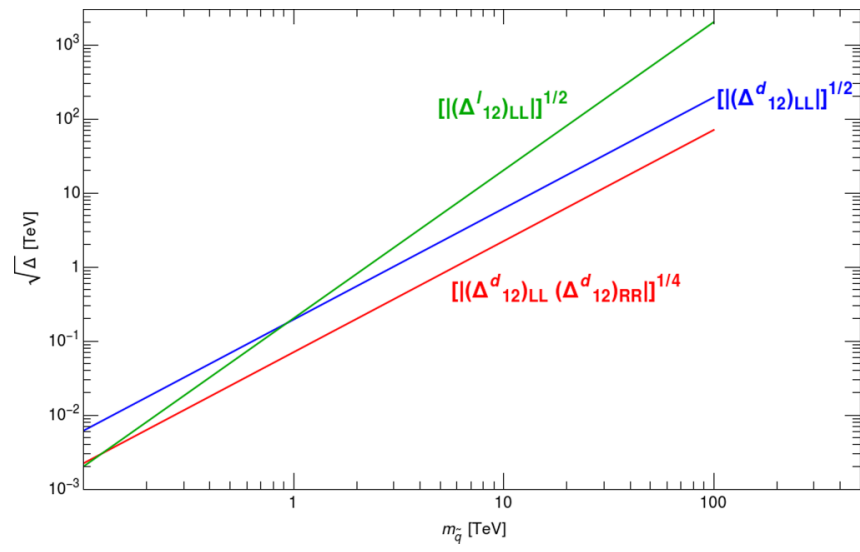

FIG. 2. Upper limits on off-diagonal squark mass terms from $\Delta m_{K}$ constraints (blue and red) and off-diagonal slepton masses from $B F(\mu \rightarrow e \gamma)$ (green).

at $90 \% \mathrm{CL}$ [56]. We plot Fig. 2 for $m_{\tilde{g}}^{2} \sim 0.3 m_{\tilde{q}}^{2}$ for $\Delta m_{K}$ constraints and $m_{\widetilde{\chi}_{1}^{0}}^{2}=0.3 m_{\tilde{\ell}}^{2}$ although the constraints only depend weakly on these mass ratios [39,40]. From Fig. 2, we see that for sfermion masses of order the weak scale $\sim 100 \mathrm{GeV}$, then the updated $\mu \rightarrow e \gamma$ branching fraction now slightly pre-empts the $\Delta m_{K}$ constraints although all require off-diagonal mass terms less than 1-10 GeV. These limits exemplify the SUSY flavor problem from days gone by when sparticles were expected to occur around the weak scale. As $m_{\tilde{f}}$ increases, then the restrictions on off-diagonal masses become increasingly mild, thus illustrating the onset of the decoupling solution to the SUSY flavor problem. For large sfermion masses, then the $\Delta m_{K}$ constraint is again most confining. For $m_{\tilde{f}} \sim 10 \mathrm{TeV}$, the off-diagonal masses are constrained to be $\lesssim 1-10 \mathrm{TeV}$, while for landscape SUSY masses, where first/second geenration sfermions are expected in the 20-30 $\mathrm{TeV}$ range, then the off-diagonal limits are $\lesssim 5-50 \mathrm{TeV}$. Such values are only mildly suppressed compared to the average squark/slepton masses although one must proceed into the $m_{\tilde{f}} \sim 100 \mathrm{TeV}$ range for unfettered flavor violation [43].

Along with limits on off-diagonal mass matrix terms, to achieve flavor universality one needs degeneracy on the diagonal. Limits on degeneracy have been computed in Misiak et al. [41]. From the $\Delta m_{K}$ constraint, for the first two generations of squarks, these amount to

$$
\left|m_{\tilde{q} 1}-m_{\tilde{q} 2}\right| \lesssim 2 m_{c} m_{\tilde{q}}^{2} / m_{W}^{2}
$$

for both up and down squarks. Thus, for sparticle masses of order $m_{W}$, splittings of only a few $\mathrm{GeV}$ are allowed and we must be in a state of near degeneracy. As $m_{\tilde{q}}$ increases, then these bounds become much weaker.

The situation is shown in Fig. 3 where we plot the GUT scale values of the first two generation sfermion masses $m_{0}(2)$ versus $m_{0}(1)$ [as $m_{0}(1,2)$ increase, then weak scale sfermion masses are nearly equal to high scale sfermion masses]. The line of degeneracy is solid black, while the bounds from Misiak et al. are labeled in green. Here, we see that for sparticle masses of order the weak scale, then rather strict degeneracy is required. However, as $m_{0}(1,2)$ increase, then 

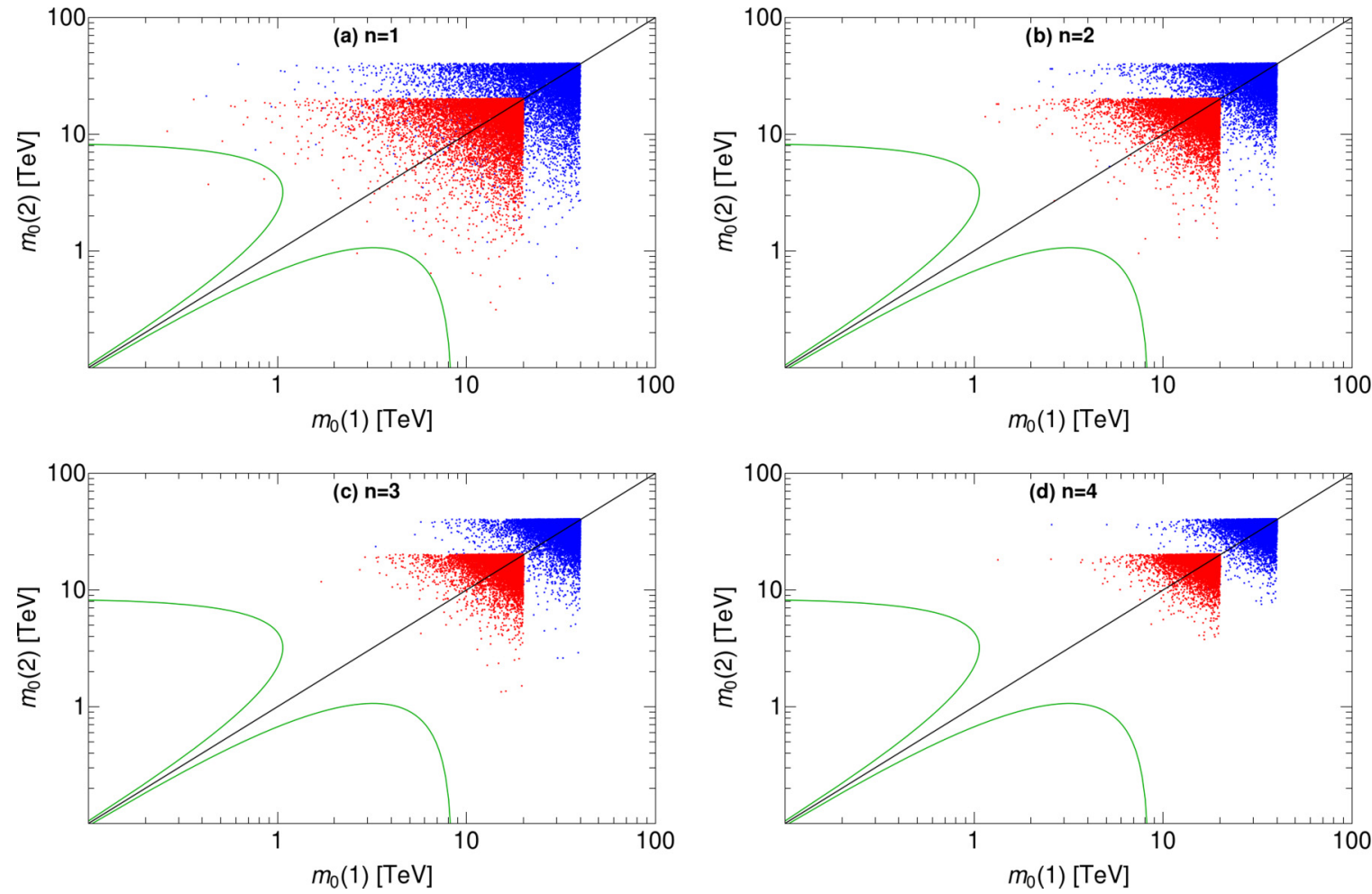

FIG. 3. The values of $m_{0}$ (2) vs $m_{0}$ (1) from an (a) $n=1$, (b) 2, (c) 3, and (d) 4, statistical selection of first and second generations matter scalar soft terms. The lower-left of green curves is excluded while red points denote soft terms scanned up to $20 \mathrm{TeV}$, while blue points show points scanned up to $40 \mathrm{TeV}$.

degeneracy is gradually relaxed until by $m_{0}(1,2) \sim 10 \mathrm{TeV}$ the bounds essentially disappear, showing again the decoupling solution. In each of the four frames, we also show the predicted landscape distribution of sfermion masses for a statistical draw of (a) $n=1$, (b) 2, (c) 3, and (d) 4 . We adopt particular, flavor-independent upper bounds of $m_{0}(1,2)<20$ and $40 \mathrm{TeV}$ since the true upper bound is parameter dependent. In frame (a) with $n=1$, just a few landscape points lie in the excluded region. As $n$ increases, then there is a stronger statistical draw towards large soft terms and the sfermion masses are drawn to flavor independent upper bounds. Thus there is also increasing degeneracy of diagonal soft breaking terms. In this sense, the landscape provides a mixed decoupling, quasidegeneracy solution to the SUSY flavor problem. For higher $n$ values, then none of the landscape points lie in the excluded region.

\section{B. $\boldsymbol{C P}$}

Limits can also be placed on complex valued soft terms due to their inducement of $C P$ violating effects on $\epsilon$ and $\epsilon^{\prime} / \epsilon$ in the kaon system and also from neutron $\left(d_{n}\right)$ and electron $\left(d_{e}\right)$ electric dipole moments (EDMs) [40,57]. The latter contribute only to LR mixing terms and are suppressed by Yukawa couplings for the first two generations so we concentrate on the former kaon constraints.

In Fig. 4, we show the constraints on the Imaginary part $\left[\left|\operatorname{Im}\left(\Delta_{12}^{d}\right)_{L L}\right|\right]^{1 / 2}$ and $\left[\left|\operatorname{Im}\left(\Delta_{12}^{d}\right)_{L L}\left(\Delta_{12}^{d}\right)_{R R}\right|\right]^{1 / 4}$ from requiring contributions to the $\epsilon$ parameter to be below its measured value. The contributions are plotted against average first/second generation squark mass for $m_{\tilde{g}}^{2} / m_{\tilde{q}}^{2}=0.3$. From the plot, we see that for weak scale sparticle masses $m_{\tilde{q}} \sim$ $100 \mathrm{GeV}$, then the $C P$ violating mass terms are required to be below about $0.5-2 \mathrm{GeV}$. However, as $m_{\tilde{q}}$ is pulled towards the landscape expected values in the tens of $\mathrm{TeV}$ range, then the $C P$-violating masses are only constrained to be $\lesssim 4-10$ $\mathrm{TeV}$ (assuming $30 \mathrm{TeV}$ squark masses). For unfettered $C P$ violating soft masses, then squark masses are required as high as $100 \mathrm{TeV}$.

From the measured value of $\epsilon^{\prime} / \epsilon$, we can also constrain $\left[\left|\operatorname{Im}\left(\Delta_{12}^{d}\right)_{L L}\right|\right]^{1 / 2}$. These results are shown in Fig. 5 versus the

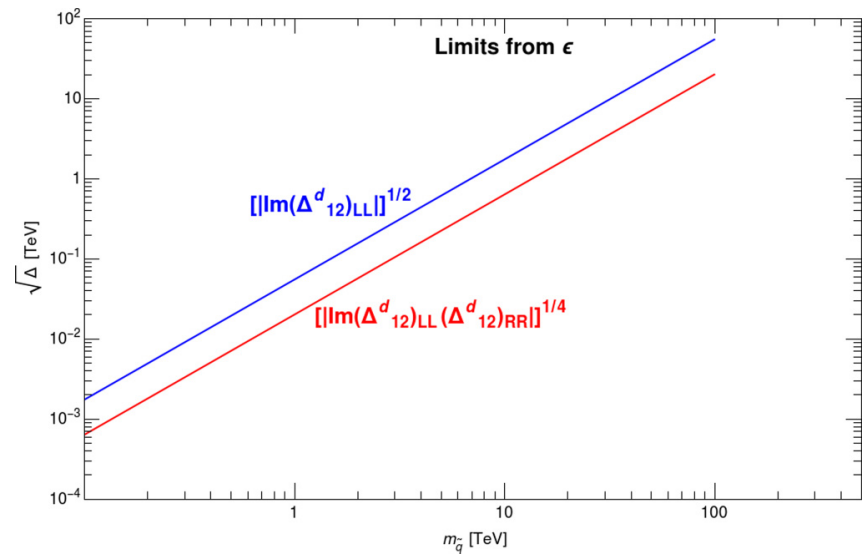

FIG. 4. Upper limits on $\left[\operatorname{Im}\left|\left(\Delta_{12}^{d}\right)_{L L}\right|\right]^{1 / 2}$ (blue) and $\left[\operatorname{Im}\left|\left(\Delta_{12}^{d}\right)_{L L}\left(\Delta_{12}^{d}\right)_{R R}\right|\right]^{1 / 4}$ (red) from kaon system $\epsilon$ constraints. 


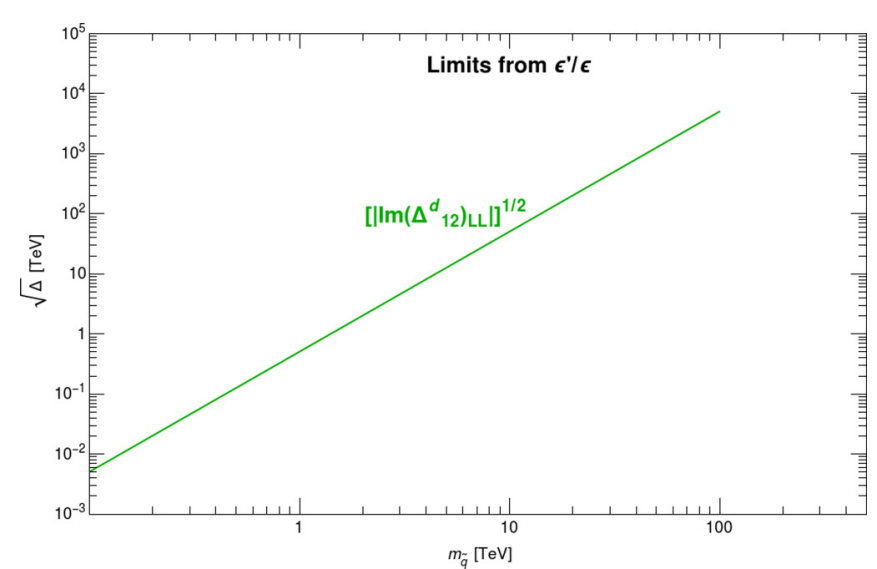

FIG. 5. Upper limits on the imaginary part of off-diagonal squark mass terms from kaon system $\epsilon^{\prime} / \epsilon$ constraints.

average first/second generation squark mass for $m_{\tilde{g}}^{2} / m_{\tilde{q}}^{2}=0.3$. For weak scale squark masses, then the $C P$-violating mass term is required to be $\lesssim 5 \mathrm{GeV}$. As $m_{\tilde{q}}$ increases into the expected landscape range of $20-40 \mathrm{TeV}$, then the $C P$-violating masses can lie in the $100 \mathrm{TeV}$ range, thus solving the SUSY $C P$ constraint at least in this channel.

\section{CONCLUSIONS}

The string theory landscape provides a compelling picture for the magnitudes of soft SUSY breaking terms. Rather general considerations of the string theory landscape from Douglas point to a statistical draw towards large soft terms while nuclear physics calculations from Agrawal et al. require values of pocket-universe weak scale value displaced by no more than a factor 2-5 from our measured value in order to produce atoms as we know them. Assuming a natural solution of the SUSY $\mu$ problem, with $\mu \sim m_{\text {weak }}$, then a statistical sampling of soft terms allows the calculation of pocket-universe $m_{\mathrm{Z}}^{\mathrm{PU}}$ via Eq. (3). The results of the statistical calculation pull $m_{h} \rightarrow 125 \pm 2 \mathrm{GeV}$ while sparticle masses are pulled beyond present LHC reach. Only higgsinos need to lie close to the weak scale.

In this paper, we focused on the landscape pull on first/second generation sfermion masses. Their upper bound does not arise from EW $D$-term contributions (which allow sfermions up to $1000 \mathrm{TeV}$ due to large, nearly perfect cancellations). Instead, their upper bound arises from two-loop RG contributions to third generation soft masses which actually push these values to small, even tachyonic values. As shown in Fig. 1, this is yet another example of the landscape pull toward living dangerously: increasing first/second generation
TABLE I. Upper bounds from various measurements of flavor changing and $C P$ violating quantities considered in text for average sfermion mass $m_{\tilde{f}}=30 \mathrm{TeV}$.

\begin{tabular}{lcc}
\hline \hline Quantity & Upper bound & Source \\
\hline$\left[\left|\left(\Delta_{12}^{d}\right)_{L L}\left(\Delta_{12}^{d}\right)_{R R}\right|\right]^{1 / 4}$ & $<12 \mathrm{TeV}$ & $\Delta m_{K}$ \\
{$\left[\left|\left(\Delta_{12}^{d}\right)_{L L}\right|\right]^{1 / 2}$} & $<30 \mathrm{TeV}$ & $\Delta m_{K}$ \\
{$\left[\left|\left(\Delta_{12}^{\ell}\right)_{L L}\right|\right]^{1 / 2}$} & $<200 \mathrm{TeV}$ & $B F(\mu \rightarrow e \gamma)$ \\
$\left|m_{\tilde{q} 1}-m_{\tilde{q} 2}\right|$ & unbounded & $\Delta m_{K}$ \\
{$\left[\left|\operatorname{Im}\left(\Delta_{12}^{d}\right)_{L L}\right|\right]^{1 / 2}$} & $<10 \mathrm{TeV}$ & $\epsilon$ \\
{$\left[\left|\operatorname{Im}\left(\Delta_{12}^{d}\right)_{L L}\left(\Delta_{12}^{d}\right)_{R R}\right|\right]^{1 / 4}$} & $<3 \mathrm{TeV}$ & $\epsilon$ \\
{$\left[\left|\operatorname{Im}\left(\Delta_{12}^{d}\right)_{L L}\right|\right]^{1 / 2}$} & $<500 \mathrm{TeV}$ & $\epsilon^{\prime} / \epsilon$ \\
\hline \hline
\end{tabular}

soft masses make the theory increasingly natural until they move it towards disallowed too large weak scale values and ultimately to CCB minima in the Higgs potential. First/second generation soft masses are thus pulled into the tens of $\mathrm{TeV}$ range towards a flavor-independent upper bound. This provides a mixed decoupling/quasi-degeneracy solution to the SUSY flavor and $C P$ problems.

We evaluated FCNC and $C P$-violating constraints in Sec. III. While the SUSY flavor and $C P$ problems do require flavor universality for weak scale sparticle masses, for sfermions in the tens of $\mathrm{TeV}$ range, then the constraints are greatly weakened but not entirely destroyed. Typically, off diagonal soft term contributions to sfermion mass matrices in the superCKM basis are required to lie in the multi-TeV region for tens of $\mathrm{TeV}$ soft terms. In addition, the pull to large, quasi-degenerate diagonal soft terms fulfills constraints on soft term degeneracy for the first two generations. Also, imaginary parts of SUSY soft terms are only mildly constrained for sfermions in the $20-40 \mathrm{TeV}$ range. As an example, we display in Table I a summary of important constraints gained for the case of average sfermion mass $m_{\tilde{f}}=30 \mathrm{TeV}$. Overall, we would conclude that the string landscape picture offers a compelling picture of at best only mild constraints on off-diagonal flavor changing soft terms and $C P$-violating masses via a mixed decoupling/quasidegeneracy solution to the SUSY flavor problem and a decoupling solution to the SUSY $C P$ problem.

\section{ACKNOWLEDGMENTS}

This work was supported in part by the US Department of Energy, Office of High Energy Physics. This paper is dedicated to the memory of Ann Nelson, whose paper on effective supersymmetry, hep-ph/9607394, was an inspiration for this work.
[1] R. Bousso and J. Polchinski, Quantization of four form fluxes and dynamical neutralization of the cosmological constant, J. High Energy Phys. 06 (2000) 006.

[2] L. Susskind, The Anthropic landscape of string theory, in Universe or Multiverse? edited by B. Carr (Cambridge University Press, 2007).
[3] M. R. Douglas and S. Kachru, Flux compactification, Rev. Mod. Phys. 79, 733 (2007).

[4] R. Bousso and J. Polchinski, The string theory landscape, Sci. Am. 291, 60 (2004).

[5] M. R. Douglas, The string theory landscape, Universe 5, 176 (2019) 
[6] A. Linde, A brief history of the multiverse, Rept. Prog. Phys. 80, 022001 (2017).

[7] F. Denef and M. R. Douglas, Distributions of flux vacua, J. High Energy Phys. 05 (2004) 072.

[8] W. Taylor and Y. N. Wang, The F-theory geometry with most flux vacua, J. High Energy Phys. 12 (2015) 164.

[9] S. Weinberg, Anthropic Bound on the Cosmological Constant, Phys. Rev. Lett. 59, 2607 (1987)

[10] S. Weinberg, The cosmological constant problem, Rev. Mod. Phys. 61, 1 (1989).

[11] L. Susskind, Supersymmetry breaking in the anthropic landscape, in From Fields to Strings: Circumnavigating Theoretical Physics, edited by M. Shifman, A. Vainshtein, and J. Wheater (World Scientific, 2005), Vol. 3, pp. 1745-1749.

[12] M. R. Douglas, Statistical analysis of the supersymmetry breaking scale, arXiv:hep-th/0405279.

[13] M. R. Douglas, The String landscape and low energy supersymmetry, arXiv:1204.6626 [hep-th].

[14] M. R. Douglas, Basic results in vacuum statistics, C. R. Phys. 5, 965 (2004).

[15] H. Baer, V. Barger, and S. Salam, Naturalness versus stringy naturalness (with implications for collider and dark matter searches, Phys. Rev. Research 1, 023001 (2019).

[16] A. Arbey, M. Battaglia, A. Djouadi, F. Mahmoudi, and J. Quevillon, Implications of a $125 \mathrm{GeV}$ Higgs for supersymmetric models, Phys. Lett. B 708, 162 (2012).

[17] P. Draper, P. Meade, M. Reece, and D. Shih, Implications of a $125 \mathrm{GeV}$ Higgs for the MSSM and low-scale SUSY breaking, Phys. Rev. D 85, 095007 (2012).

[18] H. Baer, V. Barger, and A. Mustafayev, Neutralino dark matter in mSUGRA/CMSSM with a $125 \mathrm{GeV}$ light Higgs scalar, J. High Energy Phys. 05 (2012) 091.

[19] H. Baer, V. Barger, D. Mickelson, and M. Padeffke-Kirkland, SUSY models under siege: LHC constraints and electroweak fine-tuning, Phys. Rev. D 89, 115019 (2014).

[20] H. Baer, V. Barger, P. Huang, A. Mustafayev, and X. Tata, Radiative natural SUSY with a $125 \mathrm{GeV}$ Higgs boson, Phys. Rev. Lett. 109, 161802 (2012).

[21] H. Baer, V. Barger, P. Huang, D. Mickelson, A. Mustafayev, and X. Tata, Radiative natural supersymmetry: Reconciling electroweak fine-tuning and the Higgs boson mass, Phys. Rev. D 87, 115028 (2013).

[22] S. K. Soni and H. A. Weldon, Analysis of the supersymmetry breaking induced by $\mathrm{N}=1$ supergravity theories, Phys. Lett. B 126, 215 (1983).

[23] V. S. Kaplunovsky and J. Louis, Model independent analysis of soft terms in effective supergravity and in string theory, Phys. Lett. B 306, 269 (1993).

[24] A. Brignole, L. E. Ibáñez, and C. Muñoz, Towards a theory of soft terms for the supersymmetric Standard Model, Nucl. Phys. B 422, 125 (1994); 436, 747(E) (1995).

[25] M. Dine, E. Gorbatov, and S. D. Thomas, Low energy supersymmetry from the landscape, J. High Energy Phys. 08 (2008) 098.

[26] K. J. Bae, H. Baer, V. Barger, and D. Sengupta, Revisiting the SUSY $\mu$ problem and its solutions in the LHC era, Phys. Rev. D 99, 115027 (2019).

[27] V. Agrawal, S. M. Barr, J. F. Donoghue, and D. Seckel, Anthropic considerations in multiple domain theories and the scale of electroweak symmetry breaking, Phys. Rev. Lett. 80, 1822
(1998); Viable range of the mass scale of the standard model, Phys. Rev. D 57, 5480 (1998).

[28] H. Baer, V. Barger, S. Salam, H. Serce, and K. Sinha, LHC SUSY and WIMP dark matter searches confront the string theory landscape, J. High Energy Phys. 04 (2019) 043.

[29] H. Baer, V. Barger, M. Savoy, and H. Serce, The Higgs mass and natural supersymmetric spectrum from the landscape, Phys. Lett. B 758, 113 (2016).

[30] H. Baer, V. Barger, H. Serce, and K. Sinha, Higgs and superparticle mass predictions from the landscape, J. High Energy Phys. 03 (2018) 002.

[31] N. Arkani-Hamed and S. Dimopoulos, Supersymmetric unification without low energy supersymmetry and signatures for fine-tuning at the LHC, J. High Energy Phys. 06 (2005) 073.

[32] J. R. Ellis, K. Enqvist, D. V. Nanopoulos, and F. Zwirner, Observables in low-energy superstring models, Mod. Phys. Lett. A 1, 57 (1986); R. Barbieri and G. F. Giudice, Upper bounds on supersymmetric particle masses, Nucl. Phys. B 306, 63 (1988).

[33] D. Matalliotakis and H. P. Nilles, Implications of nonuniversality of soft terms in supersymmetric grand unified theories, Nucl. Phys. B 435, 115 (1995); M. Olechowski and S. Pokorski, Electroweak symmetry breaking with nonuniversal scalar soft terms and large tan beta solutions, Phys. Lett. B 344, 201 (1995); P. Nath and R. L. Arnowitt, Nonuniversal soft SUSY breaking and dark matter, Phys. Rev. D 56, 2820 (1997); J. R. Ellis, K. A. Olive, and Y. Santoso, The MSSM parameter space with nonuniversal Higgs masses, Phys. Lett. B 539, 107 (2002); J. R. Ellis, T. Falk, K. A. Olive and Y. Santoso, Exploration of the MSSM with nonuniversal Higgs masses, Nucl. Phys. B 652, 259 (2003); H. Baer, A. Mustafayev, S. Profumo, A. Belyaev, and X. Tata, Direct, indirect and collider detection of neutralino dark matter in SUSY models with non-universal Higgs masses, J. High Energy Phys. 07 (2005) 065.

[34] H. Baer, V. Barger, and P. Huang, Hidden SUSY at the LHC: The light higgsino-world scenario and the role of a lepton collider, J. High Energy Phys. 11 (2011) 031; Z. Han, G. D. Kribs, A. Martin, and A. Menon, Hunting quasidegenerate Higgsinos, Phys. Rev. D 89, 075007 (2014); H. Baer, A. Mustafayev, and $\mathrm{X}$. Tata, Monojet plus soft dilepton signal from light higgsino pair production at LHC14, ibid. 90, 115007 (2014); C. Han, D. Kim, S. Munir, and M. Park, Accessing the core of naturalness, nearly degenerate higgsinos, at the LHC, J. High Energy Phys. 04 (2015) 132; H. Baer, V. Barger, M. Savoy, and X. Tata, Multichannel assault on natural supersymmetry at the high luminosity LHC, Phys. Rev. D 94, 035025 (2016).

[35] A. M. Sirunyan et al. (CMS Collaboration), Search for new physics in events with two soft oppositely charged leptons and missing transverse momentum in proton-proton collisions at $\sqrt{s}=13 \mathrm{TeV}$, Phys. Lett. B 782, 440 (2018).

[36] The ATLAS collaboration (ATLAS Collaboration), Searches for electroweak production of supersymmetric particles with compressed mass spectra in $\sqrt{s}=13 \mathrm{TeV} p p$ collisions with the ATLAS detector, ATLAS-CONF-2019-014.

[37] J. M. Gerard, W. Grimus, A. Raychaudhuri, and G. Zoupanos, Super Kobayashi-Maskawa CP violation, Phys. Lett. B 140, 349 (1984)

[38] F. Gabbiani and A. Masiero, FCNC in generalized supersymmetric theories, Nucl. Phys. B 322, 235 (1989). 
[39] J. S. Hagelin, S. Kelley, and T. Tanaka, Supersymmetric flavor changing neutral currents: Exact amplitudes and phenomenological analysis, Nucl. Phys. B 415, 293 (1994).

[40] F. Gabbiani, E. Gabrielli, A. Masiero, and L. Silvestrini, A Complete analysis of FCNC and $\mathrm{CP}$ constraints in general SUSY extensions of the standard model, Nucl. Phys. B 477, 321 (1996)

[41] M. Misiak, S. Pokorski, and J. Rosiek, Supersymmetry and FCNC effects, Adv. Ser. Direct. High Energy Phys. 15, 795 (1998).

[42] S. P. Martin and M. T. Vaughn, Two loop renormalization group equations for soft supersymmetry breaking couplings, Phys. Rev. D 50, 2282 (1994); 78, 039903(E) (2008).

[43] N. Arkani-Hamed and H. Murayama, Can the supersymmetric flavor problem decouple? Phys. Rev. D 56, R6733 (1997).

[44] H. Baer, C. Balazs, P. Mercadante, X. Tata, and Y. Wang, Viable supersymmetric models with an inverted scalar mass hierarchy at the GUT scale, Phys. Rev. D 63, 015011 (2001).

[45] H. Baer, C. Balazs, M. Brhlik, P. Mercadante, X. Tata, and Y. Wang, Aspects of supersymmetric models with a radiatively driven inverted mass hierarchy, Phys. Rev. D 64, 015002 (2001).

[46] A. G. Cohen, D. B. Kaplan, and A. E. Nelson, The More minimal supersymmetric standard model, Phys. Lett. B 388, 588 (1996).

[47] N. Arkani-Hamed, S. Dimopoulos, and S. Kachru, Predictive landscapes and new physics at a TeV, arXiv:hep-th/0501082.
[48] S. L. Glashow, J. Iliopoulos, and L. Maiani, Weak interactions with Lepton-Hadron symmetry, Phys. Rev. D 2, 1285 (1970).

[49] M. K. Gaillard and B. W. Lee, Rare Decay Modes of the KMesons in Gauge Theories, Phys. Rev. D 10, 897 (1974).

[50] S. Dimopoulos and D. W. Sutter, The Supersymmetric flavor problem, Nucl. Phys. B 452, 496 (1995).

[51] H. Baer and X. Tata, Weak Scale Supersymmetry: From Superfields to Scattering Events (Cambridge University Press, Cambridge, UK, 2006) p. 537; for a recent review, see, e.g., D. J. H. Chung, L. L. Everett, G. L. Kane, S. F. King, J. D. Lykken, and L. T. Wang, The Soft supersymmetry breaking Lagrangian: Theory and applications, Phys. Rept. 407, 1 (2005).

[52] J. R. Ellis and D. V. Nanopoulos, Flavor changing neutral interactions in broken supersymmetric theories, Phys. Lett. B 110, 44 (1982).

[53] J. F. Donoghue, H. P. Nilles, and D. Wyler, Flavor changes in locally supersymmetric theories, Phys. Lett. B 128, 55 (1983).

[54] M. J. Duncan and J. Trampetic, The supersymmetric QCD and $K_{L} K_{S}$ mass difference, Phys. Lett. B 134, 439 (1984).

[55] A. Bouquet, J. Kaplan, and C. A. Savoy, On flavor mixing in broken supergravity, Phys. Lett. B 148, 69 (1984).

[56] A. M. Baldini et al. (MEG Collaboration), Search for the lepton flavour violating decay $\mu^{+} \rightarrow \mathrm{e}^{+} \gamma$ with the full dataset of the MEG experiment, Eur. Phys. J. C 76, 434 (2016).

[57] M. Dugan, B. Grinstein, and L. J. Hall, CP violation in the minimal $\mathrm{N}=1$ supergravity theory, Nucl. Phys. B 255, 413 (1985). 